\section{A case of purple urine bag syndrome in an elderly patient}

Carlo Torchia, ${ }^{1}$ Raffaele Costa, ${ }^{1}$

Luigi Scalise, ${ }^{1}$ Edoardo Suraci, ${ }^{1}$

Giovanni Ruotolo ${ }^{1}$

${ }^{1}$ SOC Geriatrics, Azienda Ospedaliera

Pugliese-Ciaccio, Catanzaro; ${ }^{2}$ Center for

Cognitive Disorders and Dementia -

DSS Catanzaro, ASP Catanzaro, Italy

\begin{abstract}
Purple urine bag syndrome is a rare condition that occurs predominantly in the elderly, immobilized and bearer of chronic bladder catheter. It is a phenomenon usually associated with urinary tract infection, particularly in the presence of high bacterial load. The presence of alkaline urine, constipation, high-protein diet and dehydration are predisposing factors. In most cases it is an asymptomatic condition. We described the case of an elderly patient admitted to the geriatric Department in which purple urine was found in the urine collection bag. The culture of urine showed the presence of infection with Klebsiella pneumoniae. Targeted antibiotic treatment and proper hydration determined the resolution of the phenomenon and the resolution of the infection. Although it is an alarming phenomenon due to the particularity of the color of the urine, it is a treatable and solvable condition with an adequate specific antibiotic treatment. Prevention measures are equally effective and consist in eliminating the risk factors for this condition.
\end{abstract}

\section{Introduction}

Purple urine bag syndrome (PUBS) is a rare manifestation that occurs in elderly people with an indwelling bladder catheter (BC) and is indicative of urinary tract infection. The inspection of the urine in a catheterized patient gives us signs of immediate confirmation and of extreme diagnostic value ${ }^{1,2}$ (Table 1). Unlike traditional urinary tract infections (UTIs), those associated with PUBS are more often asymptomatic, although associated with higher bacterial loads in urinalysis. It is precisely the presence of high bacterial load that predisposes to the onset of the aforementioned syndrome, as it translates into an increase in the levels of bacterial sulfatases and phosphatases. ${ }^{3}$ We report a case of PUBS in an elderly patient with chronic BC.

\section{Case Report}

A 75-year-old man was hospitalized for the appearance of thoraco-1 pain associated with heart failure. He had a clinical history of congestive heart failure, permanent atrial fibrillation, chronic ischemic heart disease already revascularized (PCI plus stent on IVA and distal $\mathrm{Cx}$ ), type 2 diabetes mellitus, cognitive impairment (MMSE 15/30), prostate adenoma. The patient was a bearer of chronic BC due to stable urinary incontinence; the last $\mathrm{BC}$ replacement was made 4 weeks earlier. He suffered from chronic constipation and practiced the following therapy: Rivaroxaban $20 \mathrm{mg} 1 \mathrm{cp} /$ day; levosulpiride $1 \mathrm{cpx} 2 /$ day; paroxetine $1 \mathrm{cp} /$ day; metformin $10001 \mathrm{cpx} 3 /$ day; furosemide 25 mg 1 cpx2/day; spironolactone $100 \mathrm{mg} 1$ $\mathrm{cp} /$ day; bisoprolol $1.25 \mathrm{mg} 1 \mathrm{cp} /$ day; pantoprazole $20 \mathrm{mg} 1 \mathrm{cp} /$ day. The patient was apyretic and in reasonable hemodynamic compensation with acceptable vital parameters (blood pressure 135/85 $\mathrm{mmHg}$; heart rate 78 beat $/ \mathrm{min}$; oxygen saturation $97 \%$; temperature 36.5 Celsius degrees). Blood tests performed, including complete blood counts, renal function tests, liver function tests and electrolyte levels, showed leukocytosis (GB $12.30 \times 10^{3} / \mathrm{uL}$ ) with mild anemia (Hb $9.8 \mathrm{~g} / \mathrm{dL}$ ) and hyperglycemia (166 $\mathrm{mg} / \mathrm{dL}$ ), with normality of the other tests. After two days of hospitalization, purple urine was observed in the urine collection bag. Urine culture was therefore requested. The standard urine test showed urinary alkalosis ( $\mathrm{pH} 8)$, the presence of albumin (100 mg/dL), blood $(0.20 \mathrm{mg} / \mathrm{dL})$, urobilinogen $(0.2 \mathrm{mg} / \mathrm{dL})$, nitrites $(2+)$, with urine specific gravity 1018 and leukocyte esterase 500. At the examination of the sediment, rare squamous cells were highlighted, 10-20 cells per field; leukocytes $>40$ per field; several triple-phosphate crystals. The urine culture was positive for Klebsiella pneumoniae (load >1,000,000 $\mathrm{CFU} / \mathrm{mL}$ ) and, based on the antibiogram, therapy with Fosfomycin was undertaken, obtaining after two days the disappearance of the purple coloring of the urine. After three days of therapy fosfomycin $3 \mathrm{~g} /$ day, the patient was discharged with indication to add to the usual home therapy fosfomycin $3 \mathrm{~g}$ /day for another 2 days, for a total of 5 days of treatment, also in order to avoid the development of antimicrobial resistance.
Correspondence: Alberto Castagna, Center for Cognitive Disorders and Dementia DSS Catanzaro, Azienda Sanitaria Provinciale di Catanzaro, viale Crotone, 88100 Catanzaro, Italy.

Tel./Fax: +39.0961.7033013

E-mail: albertocastagna@tiscali.it

Key words: Purple urine bag syndrome; urinary infection; indoxyl sulfate; intake of tryptophan.

Contributions: RPC, AC, CT, RC, LS, ES, GR, literature research; RPC, AC, GR, manuscript writing; GR, manuscript conception; $\mathrm{AC}, \mathrm{GR}$, critical revisions.

Conflict of interest: the authors declare no potential conflict of interest.

Statement of human and animal rights: All procedures performed in studies involving human participants were in accordance with the ethical standards of the institutional and/or national research committee and with the 1964 Helsinki declaration and its later amendments or comparable ethical standards.

Informed consent: Informed consent was obtained

Received for publication: 1 March 2020. Accepted for publication: 4 June 2020.

This work is licensed under a Creative Commons Attribution-NonCommercial 4.0 International License (CC BY-NC 4.0).

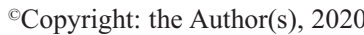

Licensee PAGEPress, Italy

Geriatric Care 2020; 6:8934

doi:10.4081/gc.2020.8934

\section{Discussion}

PUBS is a fairly rare phenomenon, first described by Barlow and Dickson in $1978 .{ }^{4}$ The subjects most exposed to this syndrome are elderly patients, chronically catheterized, suffering from chronic constipation and hypokinetic syndrome. The prevalence is greater in the female population. Furthermore, the presence of alkaline urine and the high-protein diet are predisposing factors. At the base of this condition there is a urinary infection and the pathogens that most frequently determine this phenomenon are: Pseudomonas aeruginosa, Proteus mirabilis, Morganela morganii, Escherichia coli, Providentia stuartii and $K$. pneumoniae. The pathophysiological mechanisms of purple urine staining are complex and are hypothesized to be linked to the bacterial decomposition of the tryptophan taken with the intestinal diet. In fact, in the presence of 
Table 1. Color changes in the urine as a marker of pharmacological therapy and disease. ${ }^{1,2}$

\begin{tabular}{ll} 
Color of urine & Cause \\
Milky & Lipiduria, chyluria \\
Pink & Glomerulonephritis, renal colic, propofol \\
\hline Tea & Hemolysis, myoglobinuria \\
Orange & Rifampicin \\
\hline Blue-green & Propofol, indomethacin, amitriptyline, Pseudomonas \\
Grey-black & Alkaptonuria, Addison's disease \\
\hline
\end{tabular}

Table 2. Risk factors and associated mechanism in purple urine bag syndrome.Adobe Garamond Regular

\begin{tabular}{ll} 
Risk factors for PUBS & Associated mechanism \\
Female gender & Predisposing anatomy to UTIs occurrence \\
Increased tryptophan dietary content & Increased available substrate for conversion \\
\hline Increased urine alkalinity & Facilitates indoxyl oxidation \\
Severe constipation & Increased time for bacterial deamination \\
\hline Chronic indwelling urinary catheterization & Increased risk of UTIs \\
High urinary bacterial load & Bacterial sulfatase/phosphatase availability \\
\hline Renal failure & Impaired clearance of indoxyl sulfate \\
\hline PUBS, purple urine bag syndrome; UTIs, urinary tract infections. &
\end{tabular}

sulphatase/phosphatase producing intestinal bacteria, tryptophan is transformed into indole, pyruvic acid and ammonia. These metabolites are then absorbed into the portal circulation. In the liver, the indole is conjugated in indoxyle sulfate which is filtered by the kidney and excreted in the urine, where, especially in the presence of an alkaline environment, it is transformed into indigo - which gives the color blue - and indirubin - which gives the color red. The mixture of these two colors, when urine comes into contact with the catheter tube, gives the characteristic deep purple color of the urine (Figure 1). 5,6

The interaction between the plastic of the CV tube and the catheter collection bag and the indigo and indirubin pigments, as well as the high bacterial load, are fundamental criteria for the purple urine phenomenon to occur. ${ }^{7-9}$ Dehydration is considered a risk factor for the onset of PUBS due to the increased concentration of indigo and indirubin in urine; the presence of alkaline urine is a contributing but not necessary factor, since several cases of PUBS have been described in the context of acid urine. Moreover, in patients with chronic constipation, the conversion of tryptophan to indole increases because the prolonged intestinal transit time determines an alteration of the bacterial flora. ${ }^{10}$ Therefore we can conclude that several mechanisms are implicated in the determinism of this condition, such as alterations of the metabolic pathway of the indoxyl sulfate, an increased intake of tryptophan with the diet, changes in the intestinal bacterial flora, but also chronic catheterization which determines a longer contact time between the bacterial enzymes and the indoxyl sulphate present in the urine, with consequent transformation into indigo and indirubin (Table 2). ${ }^{11}$ In

patients with chronic renal failure there is an increase in serum levels of indoxyl sulfate, therefore the presence of chronic kidney disease is considered an additional risk factor for the genesis of PUBS. ${ }^{12}$

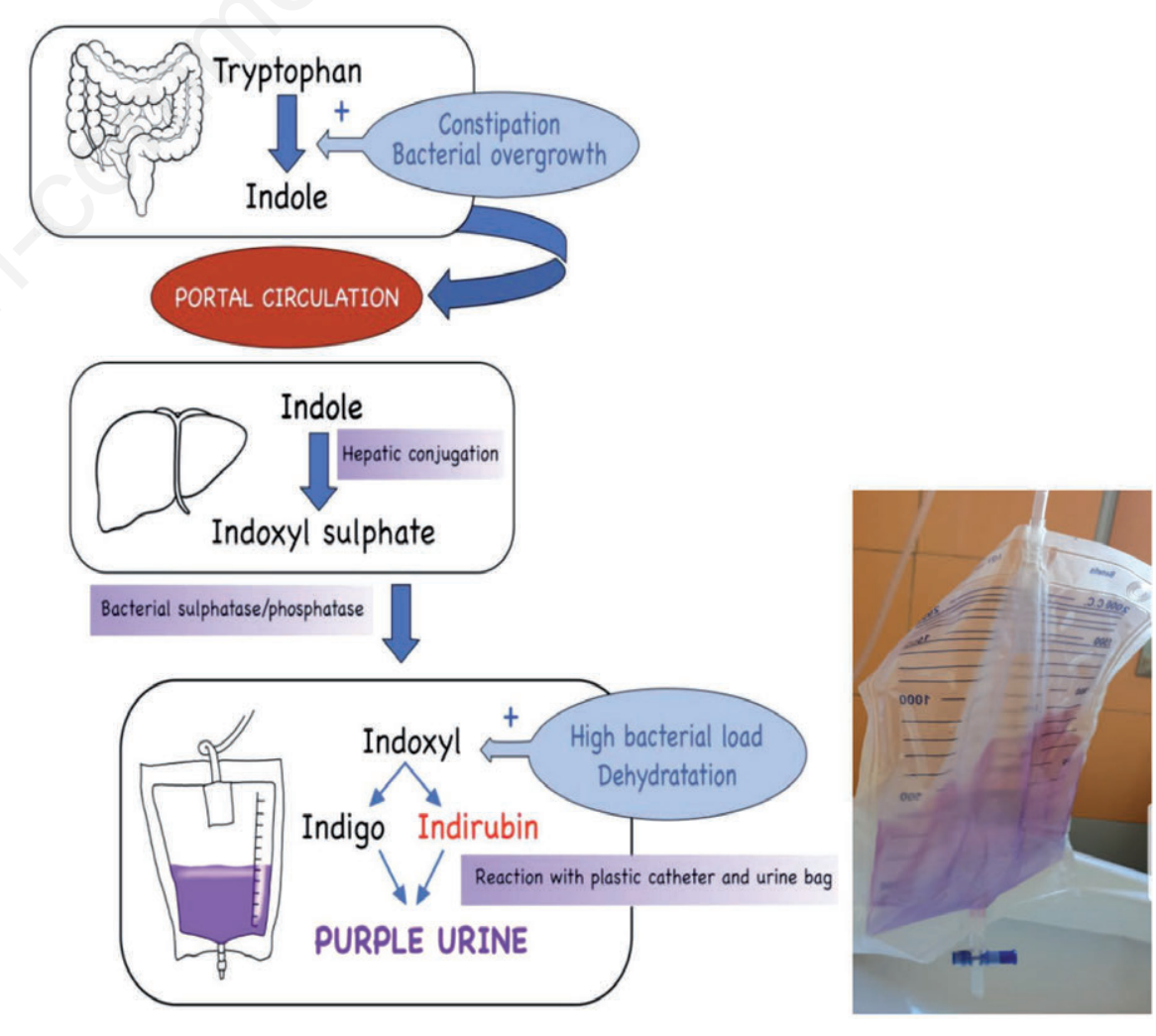

Figure 1. Physiopathological mechanism of purple urine bag syndrome. 


\section{Conclusions}

PUBS is a rare and almost always benign condition, often related to an infection and more prevalent in geriatric patients with indwelling BC. Even if this phenomenon arouses attention and often alarms the patient, his family, but also the medical and paramedical staff, in most cases it is a treatable condition, asymptomatic and often solvable with the establishment of appropriate antibiotic therapy, based on the results of urine culture. The management of PUBS, in addition to being aimed at treating urinary infection, must aim at good catheter hygiene in chronically catheterized patients, catheter replacement on schedule and constipation control. Other supportive measures, such as proper nutrition and adequate hydration, are also recommended for the prevention of recurrences. ${ }^{13,14}$

\section{References}

1. Stickler DJ. Clinical complications of urinary catheters caused by crystalline biofilms: something needs to be done. J Intern Med 2014;276:120-9.

2. Lin CH, Huang HT, Tzeng DS, et al. Purple urine bag syndrome in nursing homes: ten elderly case reports and a literature review. Clin Interv Aging 2008; 3:729-34.

3. Worku DA. Purple urine bag syndrome: An unusual but important manifestation of urinary tract infection. Case report and literature review. SAGE Open Medical Case Reports 2019;7:1-4.

4. Barlow GB, Dickson JAS. Purple urine bags. Lancet 1978;28:220-1.

5. Boentoro S, Utomo NB. Case Report: Purple urine bag syndrome in woman with neurogenic bladder. F1000Research 2019;8:979.

6. Su YJ, Yang HW. Risk factors of mortality in patients with purple urine bag syndrome. J Drug Assess 2019; 8:21-4.

7. Amoozgar B, Garala P, Velmahos VN, et al. Unilateral purple urine bag syn- drome in an elderly man with nephrostomy. Cureus 2019;11:e5435.

8. Jain N, Ramrakhiani N, Dubey MR. Purple urine bag syndrome: a striking manifestation of urinary tract infection in chronic catherised patients. Int J Sci Res 2019;8:6.

9. Wattanapisit S, Wattanapisit A, Meepuakmak A, Rakkapan P. Purple urine bag syndrome in palliative care. BMJ Support Palliat Care 2019;9:155-7.

10. Al Montasir A, Al Mustaque A. Purple urine bag syndrome. J Fam Med Primary Care 2013;2:104-5.

11. Khan F, Chaudhry MA, Qureshi N, Cowley B. Purple urine bag syndrome: an alarming hue? a brief review of the literature. Int J Nephrol 2011;2011:4192133.

12. Wang IK, Ho DR, Chang HY, et al. Purple urine bag syndrome in a hemodialysis patient. Intern Med (Tokyo, Japan) 2005;44:859-61.

13. Yang HW, Su YJ. Trends in the epidemiology of purple urine bag syndrome: A systematic review. Biomed Rep 2018;8: 249-56.

14. Plaçais L, Denier C. Purple urine after catheterization. N Engl J Med 2019;3 81:e33. 
